\title{
Peer-to-Peer MIMO Radio Channel Measurements in a Rural Area
}

\author{
Jonathan Ling, Dmitry Chizhik, Dragan Samardzija, and Reinaldo A. Valenzuela, Life Fellow, IEEE
}

\begin{abstract}
The wireless communication channel between vehicular nodes in an ad-hoc network was assessed in a comprehensive measurement and modeling effort. Nodes were equipped with roof mounted azimuthally omnidirectional antennas, and measurements were taken at both short (line-of-sight) and long ranges, at a center frequency of $2.5 \mathrm{GHz}$. The median MIMO capacity with 8 transmitters and 10 receivers $(8 \times 10)$ was found to be about five times the corresponding $1 \times 1$ SISO capacity, and three times the corresponding $1 \times 10$ SIMO capacity. Wideband soundings of such channels were conducted, where the median rms delay spread was found to be $0.6 \mu \mathrm{s}$. Measurements, spatial correlation scales, an empirical model of pathloss, and crosspolarization properties of peer-to-peer channels in a rural area are reported.
\end{abstract}

Index Terms - Antenna correlation, channel capacity, multipleinput multiple-output (MIMO), radio propagation.

\section{INTRODUCTION}

A D-HOC links are being considered for use in future communication networks to increase network connectivity both in civilian and tactical applications (e.g. [1], [2]). Essential to the design and performance of such networks is the characterization of the radio propagation channel. The most significant distinction between the cellular and peerto-peer propagation environment is that in the latter case both ends of the link are expected to be at ground level. While channel properties have been reported primarily for cellular environments, channels and models for this specific radio network (peer-to-peer MIMO wideband) have not been thoroughly addressed in the literature.

To demonstrate a real-time, mobile, networked MIMO system in a realistic tactical environment, the Defense Advanced Research Projects Agency (DARPA) has instituted a program called Mobile Network MIMO (MNM) [2] using multiple MIMO equipped vehicles to form an ad-hoc network. The radio communications were carried out at the center frequency

Manuscript received May 15, 2005; revised June 28, 2006 and January 22, 2007; accepted February 9, 2007. The associate editor coordinating the review of this paper and approving it for publication was A. Molisch. This work was supported by the IEEE. The research reported in this document/presentation was performed in connection with Contract No. W911NF-04-C-0025 with DARPA and the U.S. Army Research Laboratory. The views and conclusions contained in this document/presentations are those of the authors and should not be interpreted as presenting the official policies or position, either expresses or implied, of the U.S. Government unless so designated by other authorized documents. Citation of manufacturer's or trade names does not constitute an official endorsement or approval of the use thereof. The U.S. Government is authorized to reproduce and distribute reprints for Government purpose not withstanding any copyright notation hereon.

The authors are with Lucent Bell Laboratories, P. O. Box 07733, Crawford Hill, Holmdel, NJ 07733 USA (e-mail: \{jonling, chizhik, dragan, rav\}@alcatel-lucent.com).

Digital Object Identifier 10.1109/TWC.2007.05349.

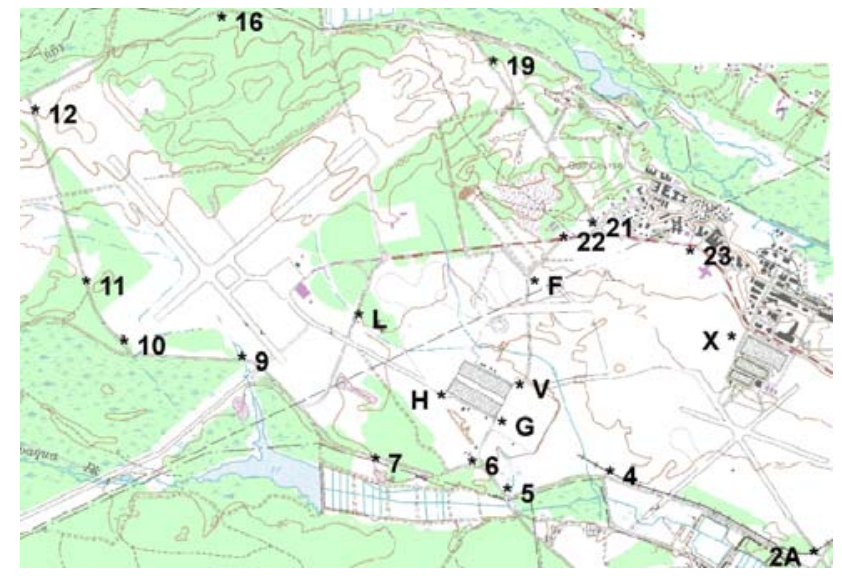

Fig. 1. Map of Lakehurst Naval Engineering Air Station with labeled points indicating receiver/transmitter locations. The diagonal distance from corner to corner of the map is about $5 \mathrm{~km}$.

of $2.5 \mathrm{GHz}$ with a bandwidth of $10 \mathrm{MHz}$. Each vehicle had 8 transmit and 10 receive antennas. The first stage of this program is to demonstrate such a system in a rural environment in Lakehurst, New Jersey.

The capacity gain for MIMO systems is especially significant in highly scattering environments, where the spatial correlation of the field is low and entries of the channel transfer matrix $\mathbf{H}$ are often assumed to be independent identically distributed (iid) complex Gaussian random variables [3]. Measurements for cellular applications have found that large MIMO capacities are supported in both urban [4], [6] and suburban [7], [8] environments with arrays of moderate size.

The Lakehurst Naval Air Engineering Station (NEAS) is located in the Pinelands of southern New Jersey. Of historical significance, it is the site of the 1937 Hindenburg crash. The area shown in Fig. 1 is approximately $3.2 \mathrm{~km}$ by $4.6 \mathrm{~km}$. Measurements were conducted between the marked locations, indicated by both numbers and letters. The diagonal extent, say from $2 \mathrm{~A}$ to 12 is $5 \mathrm{~km}$. Features include several very large hangars around points $\mathrm{H}, \mathrm{G}, \mathrm{V}$ and $\mathrm{X}$, open areas, wooded areas, runways, and mostly single story buildings. The vegetation consisted primarily of pine trees about $10 \mathrm{~m}$ in height.

\section{Measurement Equipment and Procedure}

Measurements were made separately with a $1 \times 4$ wideband sounder to determine delay spread and a $16 \times 16$ narrowband sounder to determine spatial correlation and MIMO capacity of a target $8 \times 10$ system. We first describe the wideband sounder and measurement procedure, and then the narrowband 
multi-antenna channel sounding system [4] and measurement procedure.

\section{A. Wideband Equipment and Measurement Procedure}

The wideband sounder emits a length 1023 bipolar pseudo random m-sequence occupying a $6 \mathrm{MHz}$ bandwidth centered around $2.5 \mathrm{GHz}$. Four receivers were used to amplify, downconvert, and sample the IF waveform, and digitally downconvert to baseband. The raw data was buffered and stored to be post-processed on a PC. The primary goal of these measurements was to collect pathloss and Power Delay Profiles (PDP) between different location pairs indicated in Fig. 1.

To conduct wideband measurements, the procedure consisted of placing the transmitter and receiver vehicles at selected points (Fig. 1). At each location pair, a $4 \mathrm{~W}$ signal was transmitted from a single $6 \mathrm{dBi}$ antenna and received on four antennas spaced 18 inches apart across the width of the vehicle, which included two $6 \mathrm{dBi}$ antennas and two 8 $\mathrm{dBi}$ antennas. In these wideband measurements all antennas were azimuthally omnidirectional and vertically polarized. In post processing, the time delay of the largest peak was found through circular convolution of the received signal with a replica of the transmitted sequence. A LS filter was then used to extract the impulse response, positioned to capture $10 \mu$ s of pre-cursors and $20 \mu$ s of post-cursors. Note, for the model $\mathbf{r}=\mathbf{S h}+\mathbf{n}, \mathbf{S}$ is a matrix whose rows contain shifted versions of the transmitted sequence, $\mathbf{r}$ is a column vector containing temporal samples of the received sequence, and $\mathbf{n}$ is vector representing noise. The LS estimate of the channel is simply $\widehat{\mathbf{h}}=\left(\mathbf{S}^{T} \mathbf{S}\right)^{-1} \mathbf{S}^{T} \mathbf{r}$. At each location, as the receiver vehicle was driven at $20 \mathrm{mph}$, a total of three $1 \times 4$ impulse response were collected, equally spread across 0.25 seconds, i.e. spaced about $1.2 \mathrm{~m}$. Since this spacing is larger than average correlation distance (found to be $0.7 \mathrm{~m}$ ), these twelve impulse response measurements may be considered independent. The effect of the small scale spatial fading may be reduced through incoherent (power) averaging of both the power delay profile as well as pathloss.

\section{B. Narrowband Equipment and Measurement Procedure}

Identical arrays of 16 antennas were placed on the roofs of both the transmit and receive vehicles, about $2 \mathrm{~m}$ above ground. Each array consisted of 8 vertically and 8 horizontally polarized antennas. All antennas were azimuthally omnidirectional, with the elevation gains of $8 \mathrm{dBi}$ and $9 \mathrm{dBi}$, for vertically and horizontally polarized antennas, respectively. Use of azimuthally omnidirectional antennas allows direct assessment of the impact of using dual polarization, as opposed to the use of a linear array of electric dipoles oriented horizontally which involves a change not only in polarization but of directional pattern as well.

While the narrowband MIMO sounder allows measurement of $16 \times 16$ channel responses, $8 \times 10$ MIMO capacity was of interest in this study and a subset of antennas was used to compute capacity by selecting antennas with the maximum separation. All antennas were used to estimate other channel properties, such as spatial correlations. The eight transmit antennas considered in the study consisted of 4 pairs of vertically and horizontally polarized antennas placed at the 4 corners of the $3 \mathrm{~m} \times 1.5 \mathrm{~m}$ roof of the vehicle. The 10 receive antennas used to evaluate MIMO capacity were arranged similarly on the receive vehicle, with an additional antenna pair placed at the center of the roof. The cross-polarized antennas in each pair were separated by 2 wavelengths (about $0.24 \mathrm{~m})$.

The narrowband MIMO measurement procedure consisted of radiating an $18 \mathrm{dBm} \mathrm{CW}$ signal of unique frequency from each of the sixteen transmit antennas and recording the signals received on sixteen receive antennas. The transmit signal frequencies were centered around $2.5 \mathrm{GHz}$, separated from each other by $2 \mathrm{kHz}$, with the total transmit bandwidth of 30 $\mathrm{kHz}$, which is much narrower than the coherence bandwidth of the channel. The transmit signals were separated spectrally in post processing through the use of the FFT. The result is a $16 \times 16 \mathbf{H}$ matrix containing narrowband complex channel coefficients, reported every $3 \mathrm{~ms}$. While the transmit vehicle was stationary, the receive vehicle was driven at $20 \mathrm{mph}$ during measurements so as to allow quasi-stationary channel snapshots while collecting statistically diverse $\mathbf{H}$ matrix data over longer time records.

\section{PATHLOSS AND CROSS-Polarization COUPLing}

The measured pathloss is defined as the ratio of the transmitted power and a local spatial average of the received power between two equivalent isotropic antennas. For example, when both receive and transmit antennas are vertically polarized

$$
\widetilde{L}_{\nu \nu}=10 \log _{10}\left(E_{t, n_{t}, n_{r}}\left\{\frac{P_{T} G_{T} G_{R}}{P_{R}\left(t, n_{t}, n_{r}\right)}\right\}\right)
$$

where the subscripts denote the polarization of the transmitting and receiving antenna, $P_{T}$ is the transmit power, $P_{R}$ is the received power, $G_{T}$ and $G_{R}$ are the antenna gains of the vertically polarized antennas, and the expectation is over all vertically polarized transmit receive pairs and time. For the narrowband measurement, assuming independence between locations spaced $0.7 \mathrm{~m}$, this gives 120 independent samples, and the and the $95 \%$ confidence interval is $\pm 0.08 \mathrm{~dB}$ (eg. [9]).

Use of both vertically and horizontally polarized antennas introduces additional quantities that are needed to characterize the channel. One such quantity is the difference in pathloss suffered by vertically and horizontally polarized signals. Their difference is

$$
\widetilde{L}_{\Delta}=\widetilde{L}_{\nu \nu}-\widetilde{L}_{H H}(\mathrm{~dB}) .
$$

The distribution of this quantity is plotted in Fig. 2. It may be observed that the median difference in pathlosses is quite close to $0 \mathrm{~dB}$, implying that the measured channel does not give either polarization preferential treatment. The $14 \mathrm{~dB}$ range in $\widetilde{L}_{\Delta}$ can be primarily attributed to the environment rather than measurement uncertainty due to the tight confidence interval of $\pm 0.8 \mathrm{~dB}$.

Another quantity of importance is the cross-polarization coupling present in the channel. It is a measure of the relative power penalty due to polarization mismatch, suffered by receiving the signal emitted in one polarization by an 


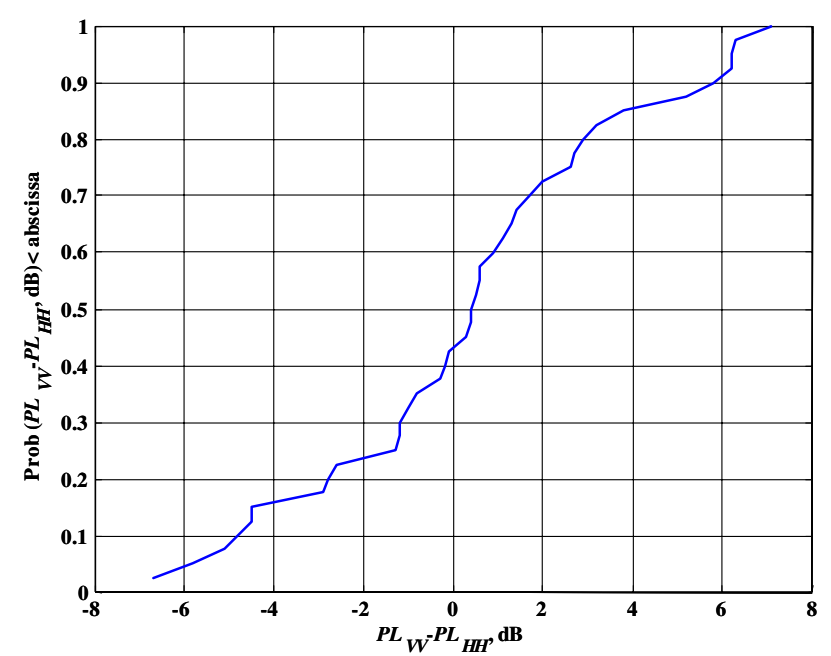

Fig. 2. Distribution of the difference in pathloss suffered by vertically and horizontally polarized signals.

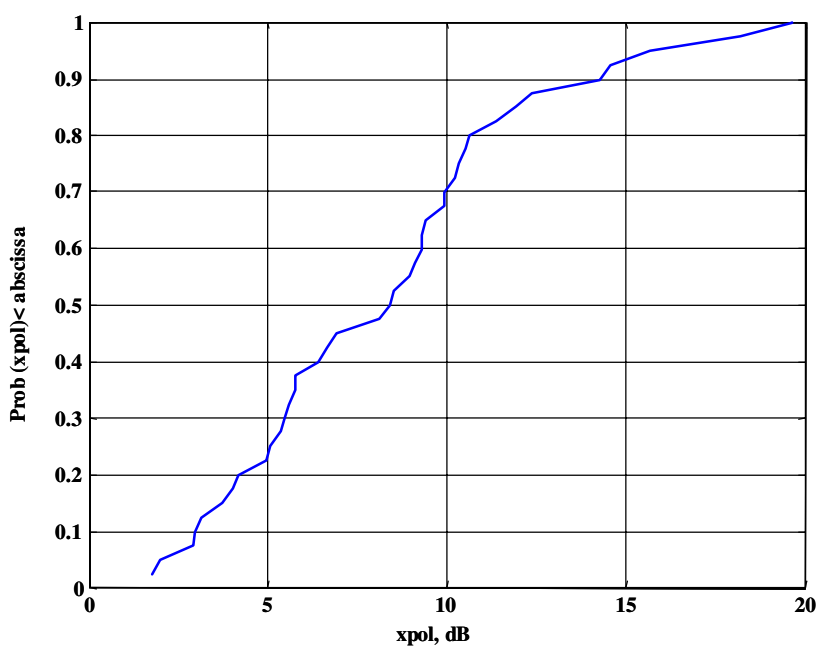

Fig. 3. Distribution of cross-polarization coupling measurements.

antenna polarized in the orthogonal polarization. The crosspolarization discrimination $x_{p o l}$ is defined by:

$$
x_{p o l}=\left(L_{V H}+L_{H V}\right) / 2-\left(L_{V V}+L_{H H}\right) / 2(\mathrm{~dB}) .
$$

The distribution of $x_{p o l}$ is plotted in Fig. 3. Cross-polarization discrimination was observed to vary from 2 to $19 \mathrm{~dB}$ with a median of $8.5 \mathrm{~dB}$, which is slightly higher than $6 \mathrm{~dB}$ reported in urban channels [10]. Perhaps due to extensive spatial averaging, no negative values of $x_{p o l}$ were measured as reported in [11]. When both horizontally and vertically polarized antennas are used at the transmitter and at the receiver, the narrowband received signal vector $\mathbf{r}$ in the presence of receive noise vector $\mathbf{n}$ is related to the transmitted signal vector $\mathbf{s}$ through:

$$
\mathbf{r}=\left(\begin{array}{ll}
\mathbf{H}_{V V} & \mathbf{H}_{V H} \\
\mathbf{H}_{H V} & \mathbf{H}_{H H}
\end{array}\right) \mathbf{s}+\mathbf{n}
$$

where the channel $\mathbf{H}$-matrix is written in terms of block matrices corresponding to different polarizations of receive and transmit antennas, as indicated by subscripts. The entries of the block matrices have the average power given by $E\left\{\left|\mathbf{H}_{V V}\right|_{n m}^{2}\right\}=10^{-0.1 L}, E\left\{\left|\mathbf{H}_{H H}\right|_{n m}^{2}\right\}=10^{-0.1 L}$,

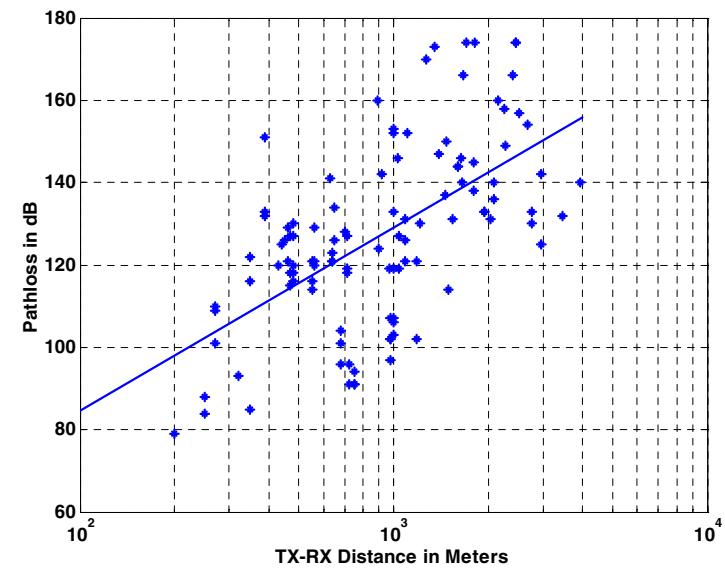

Fig. 4. Measured pathloss with regression line.

$E\left\{\left|\mathbf{H}_{V H}\right|_{n m}^{2}\right\}=10^{-0.1\left(L-x_{p o l}\right)}$, and $E\left\{\left|\mathbf{H}_{H V}\right|_{n m}^{2}\right\}=$ $10^{-0.1\left(L-x_{p o l}\right)}$, where the co-polarized pathloss $L \approx L_{V V} \approx$ $L_{H H}$, an approximation following from generally small difference in pathoss (2) observed in Fig. 2.

Given particular (measured) values of co-polarized pathloss $L$, large values of $x_{p o l}$ result in a reduction in total received power, as a particular receive antenna will receive weaker signals arriving from the orthogonally polarized transmit antennas. It is known that cross-polarized signals are largely uncorrelated [10]. So in the context of MIMO, the use of both polarizations increases the statistical independence of the transmitted streams which is beneficial, but reduces the total received power, which is detrimental. For example, for two arrays with an equal number of horizontally and vertically polarized antennas, an infinite value of $x_{p o l}$ leads to a maximum received power reduction of $3 \mathrm{~dB}$, as half of the entries of the channel H-matrix in (4) would have zero power. Use of both polarizations in a non-scattering environment, such as in freespace, allows two spatially multiplexed channels, as opposed to only spatial channel available in single polarization.

Measured co-polarized pathloss $L$, including data from both wideband and narrowband measurements, is plotted as a function of transmitter-receiver separation $d$ in Fig. 4. The data includes both LOS and NLOS links, with LOS comprising about $10 \%$ of the data. Also plotted is the least mean square regression line $\widetilde{L}(d)$, which represents pathloss in $\mathrm{dB}$ as a function of distance $d$ in meters:

$$
\widetilde{L}(d)=129+44 \log _{10}(d / 1000)(\mathrm{dB}) .
$$

Using [9] the standard deviation of the regression coefficients is $1.7 \mathrm{~dB}$ in the intercept, and 5.7 in the slope. The standard deviation of error of the regression fit was found to be $17.5 \mathrm{~dB}$, which corresponds to the shadow fading standard deviation. The relatively large shadow fading deviation may be attributed in part to the heterogeneity of the environment, which included large open areas, wooded areas, and areas with large obstructions, such as hangars, which become more significant with low antenna height. For comparison, in a cellular environment characterized by a tall base station, a representative median pathloss for suburban level terrain with 
moderate to heavy tree density is $120 \mathrm{~dB}$ at $1 \mathrm{~km}$, accompanied by $9 \mathrm{~dB}$ shadow fading standard deviation [12].

\section{FReQuency Selectivity}

A total of 45 links were used to collect power delay profiles, based on 12 impulse responses at each link as described in Section 2.1. Three of these links may be classified as Line of Sight (LOS). Measures of delay spread such as rms delay spread, and $90^{\text {th }}$ and $95^{\text {th }}$ percentile energy support were computed from the spatially averaged power delay profiles. The time spanned by the significant measured arrivals, whose total energy was ninety percent of the energy of the measured power delay profile is defined as $90^{\text {th }}$ percentile energy support. For all measures significant arrivals are defined as having power at least $5 \mathrm{~dB}$ above the average noise level. This sets the probability of a false positive (Type I error) to 0.001 . The $95 \%$ confidence interval for the mean power is $(-1.9 \mathrm{~dB},+1.6 \mathrm{~dB})$. The rms delay spread is defined as:

$$
\widetilde{\tau}_{r m s}=\sqrt{\frac{\sum_{k=1}^{K}\left|\tau_{k}-\tau_{m}\right|^{2} p_{k}}{\sum_{k=1}^{K} p_{k}}}, \quad \tau_{m}=\frac{\sum_{k=1}^{K} \tau_{k} p_{k}}{\sum_{k=1}^{K} p_{k}}
$$

where $p_{k}$ is the power of the received signal at the $\tau_{k}$-th delay for a measurement at a particular location.

Defining the cumulative sum of the normalized power delay profile as

$$
F_{\tau}(\tau)=\frac{\sum_{\tau_{k}=0}^{\tau} p_{k}}{\sum_{\tau_{k}=0}^{\infty} p_{k}}
$$

the $90^{\text {th }}$ and $95^{\text {th }}$ percentile energy support may be defined in terms of the inverse of (7):

$$
\begin{aligned}
\tau_{90} & =F_{\tau}^{-1}(0.90) \\
\tau_{95} & =F_{\tau}^{-1}(0.95)
\end{aligned}
$$

A cumulative distribution of these measures of delay spread is plotted in Fig. 5. It may be observed that the median and $90^{\text {th }}$ percent $\mathrm{rms}$ delays are $0.6 \mu \mathrm{s}$ and $1.6 \mu \mathrm{s}$, respectively. Our findings are slightly higher than the median rms delays for high base to mobile measurements in suburban and rural areas reported to range from 0.1 to $0.25 \mu \mathrm{s}$ [13]-[16]. This may be caused by scattering from the four hangars which are large structures, formerly housing trans-Atlantic dirigibles. Although these structures are quite unusual, delay spreads are not out of line as compared to suburban Toronto where the $90^{\text {th }}$ percent rms delay was reported as $1.2 \mu \mathrm{s}$ [16]. We conclude that delay spread for peer-to-peer ground level nodes in not significantly altered as compared to a high base positioned over the clutter. One admittedly speculative interpretation of this is that lowering the base antenna into the clutter affects all delays equally.

It may be noted that for locations around the hangars which corresponded primarily to open areas, the pathloss was generally rather low, with free space loss being a good

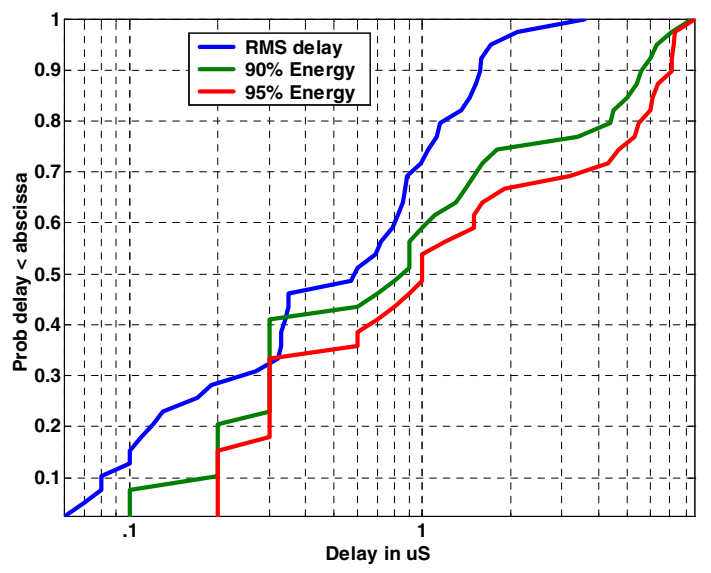

Fig. 5. CDF of rms delay spread, $90^{\text {th }} \& 95^{\text {th }}$ percentile energy support.

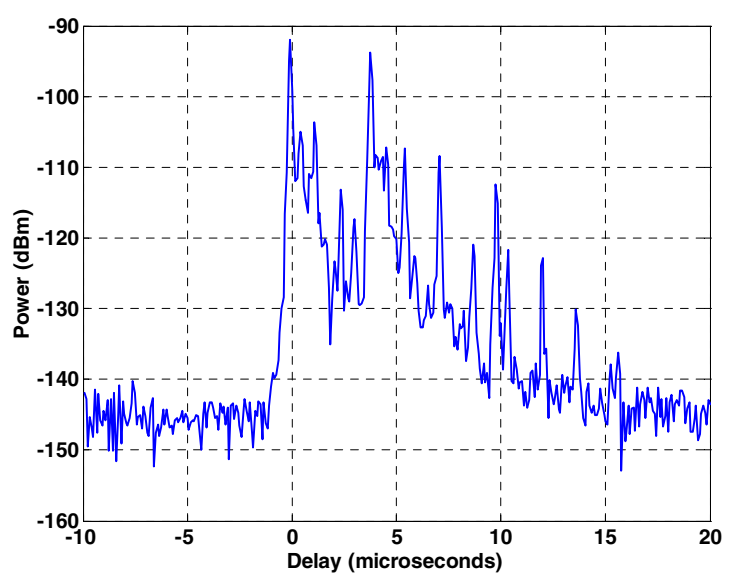

Fig. 6. Delay profile from non-LOS link (23), (22) with very high delay spread $(2.1 \mu \mathrm{s}) .125 \mathrm{~dB}$ pathloss.

approximation, and the delay spreads were also very low. Higher pathloss accompanied by larger delay spreads were characteristic of links with direct path blocked by a hangar or trees. It may be noted that the relative importance of signal components scattered from large distant objects (such as hangars in open terrain) increases once the direct path is blocked. The rms delay spread varied from $0.07 \mu \mathrm{s}$ in the parking lot (at about the resolution limit of the $6 \mathrm{MHz}$ sounding waveform) to $2.1 \mu$ s measured on the obstructed (23, 22) link, Fig. 6. Note that here and in the rest of the paper the pair label (e.g. $(23,22))$ corresponds to the transmitter, receiver locations, respectively.

Both the $90^{\text {th }}$ and $95^{\text {th }}$ percent energy support reported in Fig. 5 may give an impression of excessive frequency selectivity. A more direct measure of frequency selectivity is a frequency autocorrelation function $R\left(f_{c}, f_{c}+f\right)$, which is the correlation between the channel response at $f_{c}$ and $f_{c}+f$ [17]. Since it gives a measure of the channel 'flatness,' it may be helpful in determining the width of OFDM sub-bands. This quantity is plotted for several selected links in Fig. 7. 


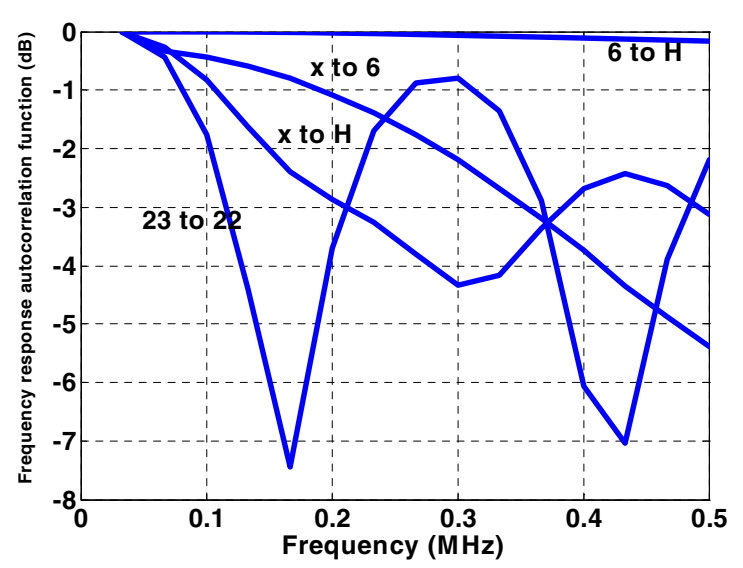

Fig. 7. Frequency autocorrelation for four selected locations.

\section{NARROWBAND MIMO CAPACITY}

MIMO capacity is measured directly here, though it may be inferred from angle spread or correlation measurements on both ends, as was reported in the double directional channel measurements in [18] and [19]. The direct measurement ensures that degenerate channels such as keyholes or pinholes [20], [21] are not ruled out a priori. This spatial degeneracy occurs when, for example, all paths are diffracted over a single wedge.

In the case of the channel that is unknown at the transmitter, the capacity of the MIMO system with $n_{t}$ transmit antennas and $n_{r}$ receive antennas is given by the formula [3]:

$$
C(\rho)=\log _{2}\left|\mathbf{I}_{n_{r}}+\left(\frac{\rho}{n_{t}}\right) \mathbf{H} \mathbf{H}^{\dagger}\right| \mathrm{bps} / \mathrm{Hz}
$$

where the total transmit power $P_{T}$ is equally allocated among the transmit antennas. We define the average SNR, also called the system SNR, as $\rho=P_{r} / N_{o}$, where $P_{r}$ is the average received power. $\mathbf{H}$ is the matrix of complex channel coefficients, normalized to have unit average power, $N_{o}$ is the power of AWGN, $\mathbf{I}_{n_{r}}$ is the identity matrix of dimension $n_{r}$, and $\dagger$ designates the complex conjugate transpose.

\section{A. Long Range MIMO Capacity}

Long range dual polarized measurements consisted of forty links, with five of these links classified as Line of Sight (LOS). At any one link, the transmitter was stationary while the receiver was moving at $20 \mathrm{mph}$ over 2 meters, recording 70 $16 \times 16 \mathbf{H}$ matrices. It was found for such data segments that large scale characteristics such as pathloss, Doppler spectra and MIMO capacity did not change significantly during such translation.

The capacity evaluated at a fixed system SNR is a measure of scattering richness of a MIMO channel. An arguably better metric of system performance may be capacity evaluated at the expected operational SNR. This would account for different propagation losses suffered on different links. The expected operational SNR may be estimated using measured pathloss $L$ :

$\rho_{o p}=\min \left[\begin{array}{c}\left(P_{T}+G_{T}+G_{R}-L(d)-\right. \\ \left.L_{\text {cable }_{T}}-L_{\text {cable }_{R}}-10 \log _{10}(F k T B)\right), 23\end{array}\right] \mathrm{dB}$ where $k$ is the Boltzmann constant and $T$ is the effective noise temperature, set at $300^{\circ} \mathrm{K}$. It is assumed here that the noise figure $F=2$, bandwidth $B=10 \mathrm{MHz}$, total power $P_{T}$ transmitted from all antennas is $43 \mathrm{dBm}(20 \mathrm{~W})$, receiver and transmitter antennas gains $G_{T}=G_{R}=8 \mathrm{~dB}$, and receiver and transmitter cable losses are $L_{\mathrm{cable}_{T}}-L_{\mathrm{cable}_{R}}=3 \mathrm{~dB}$. The maximum effective SNR is often limited by effects such as transmitter non-linearities, phase noise, etc. For the purposes of computing capacities here, the maximum effective SNR is taken here to be $23 \mathrm{~dB}$. An estimate of the average capacity of the channel is defined as the average of the measured capacities.

$$
\widetilde{C}_{8 \times 10}\left(\rho_{o p}\right)=E_{\mathbf{H}}\left\{C_{8 \times 10}\left(\rho_{o p}\right)\right\} .
$$

For comparison, the average SISO capacity for a Rayleigh channel is defined as:

$$
\bar{C}_{S I S O}\left(\rho_{o p}\right)=\frac{1}{\rho_{o p}} \int_{0}^{\infty} \log _{2}(1+x) e^{-x / \rho_{o p}} d x
$$

where $\rho_{o p}$ is defined in (10). Such comparison allows for the same total transmit power in both SISO and MIMO cases. MIMO system is allowed both the advantage of collecting power on multiple receive antennas, as well as possible gain from spatial multiplexing. The distribution over all forty locations of locally averaged capacities using the dually polarized $8 \times 10$ array is compared against corresponding SISO capacities in Fig. 8. It may be observed that average $8 \times 10$ MIMO capacities are nearly five times the average capacities achieved by a SISO system under the same total transmit power and same average path loss. Also included is the average capacity of a $1 \times 10$ which collects the same average power as the $8 \times 10$ system, but does not permit spatial multiplexing.

There were channels where MIMO processing offers only a modest gain. One such place was on a long straight road with trees on both sides (11), (12), where the $8 \times 10$ capacity was found to be only about 3.5 times the corresponding SISO capacity. As the MIMO arrays used both polarizations, thus promising two communication modes even in free space conditions, this points to particularly poor scattering. Here the geometry suggests guiding of the radio waves such that they are received from a very narrow angle, while higher order reflections are absorbed. An analogy may be made to a waveguide with rough, lossy walls, where the high order modes decay quickly, reducing the capacity [20], [23]. An analysis of the Doppler spectrum (Section 6) confirmed that the arriving waves are indeed confined to a narrow angle.

\section{B. Short Range LOS MIMO Capacity}

Short-range narrowband measurements were collected with the vehicles driving at distances of 50 to 150 meters from each other, mostly within line of sight of each other. The vehicle pair traversed most of the area of shown in Fig. 1, resulting in a data set with hundreds of thousands of $\mathbf{H}$-matrices collected over a wide variation of both wooded and open environments. The issue for MIMO systems here is whether there is strong enough scattering in this LOS environment. A $8 \times 8$ subset of all vertical antennas was compared to a subset of $8 \times 8$ dual polarized antennas. Due to the low pathloss experienced in 


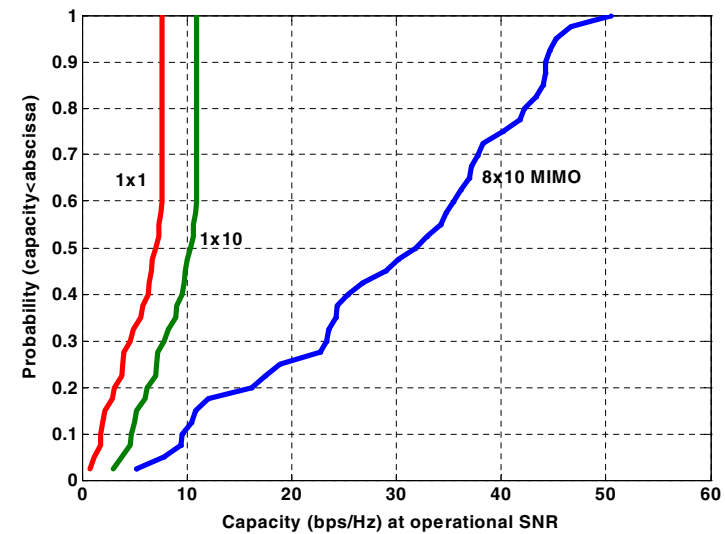

Fig. 8. Capacities of measured channels for $1 \times 1,1 \times 10$, and $8 \times 10$ systems at $S N R=\rho_{\text {op }}$.

these channels, capacity is evaluated at the maximum effective $\mathrm{SNR}$, here set at $23 \mathrm{~dB}$. It was found in this environment of low to moderate scattering and high SNR, that using the dual polarized array increases the median capacity by $30 \%$, from 32 to $42 \mathrm{bps} / \mathrm{Hz}$, over using only vertically polarized antennas. The SISO capacity is $6.7 \mathrm{bps} / \mathrm{Hz}$.

\section{Doppler And ANgular SPectra}

The connection between the angular spectrum and the Doppler spectrum experienced by a moving receiver is well established (e.g. [28]) and has been used to deduce angular spectrum properties from measured Doppler spectra (e.g [14], [30]). The relationship between the angle of arrival and the corresponding Doppler frequency is found by observing that a plane wave arriving at the mobile antenna at an angle $\alpha$ from the direction of motion will experience a Doppler frequency shift of $f_{d}=(\nu \cos \alpha) / \lambda$, where $v$ is the mobile speed and $\lambda$ denotes the wavelength. A uniform angular distribution in azimuth results in the Clark spectrum [29]. A moving mobile antenna may be considered as forming a synthetic linear aperture, with the Doppler spectrum that corresponds to beamforming measurements over this aperture. Use of a synthetic aperture formed by a single moving antenna has advantages over a comparable array formed by multiple antennas in that there is no need to calibrate the relative array responses. One limitation of the synthetic aperture is that the scatterers need to remain stationary while the antenna is traversing the length of the aperture. A linear aperture is known to have conical ambiguity, (e.g. [14]), which presents difficulties in inverting the Doppler spectrum to deduce the underlying angular spectrum. When the spectrum is confined to the azimuthal plane, such ambiguity is often called the leftright ambiguity, as it is impossible to determine whether the waves are arriving from left or right. Nevertheless it is of interest here to determine the extent of angular distribution of power at the mobile in the rural peer-to-peer environment. It is also of interest to compare the Doppler spectra observed on different polarizations.

In this work, as there are 8 antennas of each polarization at each end of the link, there are $8 \times 8=64$ measurements of fluctuating time series for each combination of
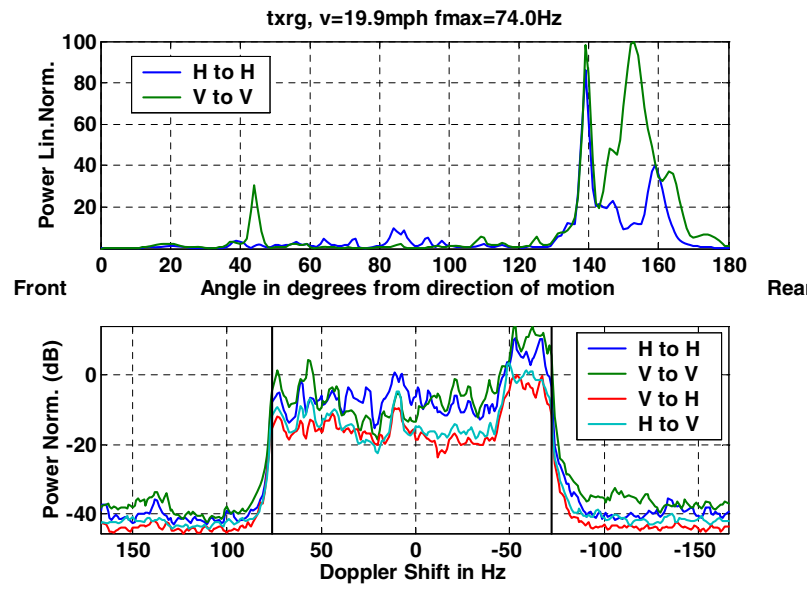

Fig. 9. Measured Doppler and corresponding angular spectra for the $(\mathrm{X}, \mathrm{G})$ link, with high capacity.

transmit/receive polarizations. The power of the raw Fourier transforms of such series are averaged to obtain an estimate of the Doppler spectrum, from which a (non-unique) angular spectrum may be deduced. When the arriving plane waves are distributed within a small range of elevation angles, rather than confined to the horizontal plane, the Doppler spectrum does not approach infinity at $\pm f \max$ [24] as in Clark's model. The angular resolution is determined from the length of the synthetic aperture, and the time windowing function. For example, using a $9 \mathrm{~m}$ synthetic aperture and a BlackmanHarris window gives full width half power resolution ranging from 2.5 degrees broadside to 4 degrees close to endfire. The maximum size of the aperture is limited by the coherence scale of the large-scale properties of the channel.

An example of both the Doppler and angular spectra is shown in Fig. 9 The total power of each spectrum was left unnormalized so as to allow comparisons of relative powers as well as angular distributions. It was found that both pathloss and capacity of the channel at this particular location varied little over $9 \mathrm{~m}$, allowing the use of $9 \mathrm{~m}$ aperture for Doppler spectrum estimation. At other locations smaller apertures were used so as to remain within the large-scale coherence scale of the channel. Note that the angular spectra in the top plot are displayed using linear relative power, while the Doppler spectra in the bottom plot are in $\mathrm{dB}$. The measured Doppler and angular spectra for the $(X, G)$ link are shown in Fig. 9 and are representative of locations with reasonably high angle spread and high capacity, $50 \mathrm{bps} / \mathrm{Hz}$ at $23 \mathrm{~dB}$ SNR, which may be compared to $57 \mathrm{bps} / \mathrm{Hz}$ achievable in an iid channel. Large peaks, observed around 140 to 160 degrees, are consistent with scattering from the hangar close to the receiver. Uniform scattering is observed about $15 \mathrm{~dB}$ below the main peaks.

Doppler and angular power spectra observed on different polarizations, with few exceptions (e.g. Fig. 9), were found to be quite similar. In such cases it may be claimed that the scatterers are not particularly selective with regard to polarization. This may be exploited to simplify modeling by assuming the same power angular spectra, and thus spatial correlations, for all polarizations as is done in Section XII.

The lower angle spread gives rise to the larger correlation 
distances discussed in Section XII. In contrast, small correlation distances [4] and wide angle spreads [26] have been reported at the mobile in urban areas. Wide angle spreads have been also predicted by ray tracing [27] in urban areas. Nevertheless we find that even in these rural areas large gains in capacity may be achieved through the use of multiple antennas, albeit with $10 \lambda$ separation between copolarized antennas.

\section{MIMO CHANNEL MODEL}

To develop a spatial model for long range communications between receive and transmit arrays, spatial correlations were deduced from data. As the antennas are arranged in the horizontal plane (on the roof of the vehicle), the relative antenna displacement may be expressed in terms of separation $x$ along the vehicle and $y$ perpendicular to the vehicle To represent the correlations compactly, it was decided to model the correlation $\Phi(x, y)$ as decaying exponentially with antenna separation within the receive and transmit arrays at each terminal :

$$
\Phi(x, y)=e^{-\alpha_{x}|x|} e^{-\alpha_{y}|y|}
$$

where the decay constants $\alpha_{x}$ and $\alpha_{y}$ are determined through fitting to correlation coefficients estimated from data. The use of exponential shape is rather arbitrary, but it was found to be an effective way to model capacity both in this work as well as in urban environment [4]. An explicit dependence on $x$ and $y$ was used in (13) to allow for possible anisotropy in the field, perhaps arising from guiding by the trees along the road. A complete correlation matrix of, say, a receiver array may be generated using representation (13) for any two antennas, separated by $x$ meters along the vehicle and $y$ meters across the vehicle. To test the adequacy of this representation, an ensemble of narrowband synthetic $\mathbf{H}$-matrices was generated by imposing a spatial correlation on an ensemble of matrices of iid complex Gaussian channel coefficients $\mathbf{H}_{i i d}$ using the separable (sometimes called Kronecker) model [28], [31]:

$$
\mathbf{H}=\boldsymbol{\Phi}_{R}^{1 / 2} \mathbf{H}_{i i d} \boldsymbol{\Phi}_{T}^{1 / 2}
$$

where the receiver correlation matrix $\boldsymbol{\Phi}_{R}$ and the transmitter correlation $\boldsymbol{\Phi}_{T}$ may be computed for the desired antenna configuration. A median capacity of an ensemble of such synthetic $\mathbf{H}$-matrices may be compared to the corresponding capacity computed from measured channel coefficients. Correlation coefficients between various antenna elements were computed for antennas displaced along and across the vehicle with $4 \lambda, 8 \lambda$, and $12 \lambda$ separations $(\lambda=0.12 \mathrm{~m}$ at $2.5 \mathrm{GHz})$. A correlation coefficient between two transmit antennas is estimated from the data as:

$$
\boldsymbol{\Phi} T_{1,2}=\frac{E\left\{h_{n, t 1} h_{n, t, 2}^{*}\right\}}{\sqrt{E\left\{\left|h_{n, t 1}\right|^{2}\right\} E\left\{\left|h_{n, t 2}\right|^{2}\right\}}}
$$

where the expectation is over all receivers, and over time as the vehicle was driven a distance of about $2 \mathrm{~m}$. Over this short distance, local average pathloss varied less than $2 \mathrm{~dB}$, and capacity computed from instantaneous $\mathbf{H}$-matrices varied less than $5 \%$. Similar processing is done for receive antennas. To determine the correlation coefficients as a function of antenna spacing and disposition (i.e. along or across the array), the amplitudes of appropriate correlation coefficients are averaged for comparably spaced transmit antennas. Correlation decay constants defined in (13) were determined for a best fit to correlations estimated as in (15). Over the entire data set, the median correlation decay constant was found to be about 0.7 $\mathrm{m}^{-1}$ (corresponding to about $1.5 \mathrm{~m}$ coherence length, where the correlation drops to $1 / e$ ), with $20 \%$ of links having decay constants of $0.1 \mathrm{~m}^{-1}$ or lower, resulting in lower capacity The number of independent measurements of correlation between 2 transmit antennas used to estimate (15) is about 24, accounting for 4 widely separated receiver antennas, 3 locations separated by the coherence length along the drive route and 2 pairs of transmit antennas at a particular spacing.

Two effects are prominent in reducing the accuracy of capacity prediction. One is the case where the model assumed in (14) is correct but the correlation parameters were estimated with error. This may occur, for example, in cases where there are not enough independent samples used to estimate channel response correlations. Another source of inaccuracy is where the model itself is incorrect, e.g. the case where the channel correlations are not separable into a product of transmitter correlation and receiver correlation as well as channels that are not Gaussian-distributed at all, as in the case of a keyhole [20] or pinhole channel [21]. In the case of a keyhole or pinhole channel spatial correlation may be low, but the channel capacity is still low. One example of this has been observed experimentally in MIMO measurements in Manhattan [4], as well as indoors in hallways [5]. Here use of the separable correlation model (14) leads to an overprediction of channel capacity, as the effective rank of the matrix is constrained not through correlations alone but also through capacity pinching. On the other hand, non-separable channel correlations may lead to ergodic capacities that are higher than capacities of iid Gaussian channels [36]. In this work it has been found that in $70 \%$ of all links and $80 \%$ of non-LOS links, the average capacity predicted using the separable correlation model (14) is larger than the locally averaged capacity directly computed from measurements, although usually by a small amount.

Excluding the LOS links which are clearly non-separable, the median error between averaged predicted and measured capacities is $3 \%$. When both LOS and non-LOS links are considered, $90 \%$ of the errors are still within 10\%. Similar accuracy was reported using a separable correlation model in an urban cellular environment in [4]. Within the reported accuracy, the separable (Kronecker) channel model (14) is judged adequate for non-LOS channels measured in this work. For reference, representing the channel coefficients as a set of iid complex Gaussian random variables would result in a $20 \%$ error in computed capacity. When the separable model was applied to short range LOS channels the error between measured and predicted capacity based on (14) and (15) would reach at worst $25 \%$. In related work [35], it was reported that using a separable channel model such as (14) based on correlations estimated from wideband indoor measurements leads to an under-prediction of channel capacity. This has been attributed to the shortcoming of the separable model

Equation (7) may be generalized to allow simulation of wideband dual polarization MIMO channel matrices. Now for 
each delay spatial correlations are applied:

$$
\begin{aligned}
\mathbf{H}(\tau) & =\sum_{k} \sqrt{P_{k} G_{p} G_{T} G_{R}} \\
& \left(\begin{array}{cc}
\boldsymbol{\Phi}_{R_{V V}}^{1 / 2} \mathbf{H}_{1}\left(\tau_{k}\right) \boldsymbol{\Phi}_{T_{V V}}^{1 / 2} & \sqrt{\eta} \boldsymbol{\Phi}_{R_{V H}}^{1 / 2} \mathbf{H}_{2}\left(\tau_{k}\right) \boldsymbol{\Phi}_{T_{V H}}^{1 / 2} \\
\sqrt{\eta} \boldsymbol{\Phi}_{R_{H V}}^{1 / 2} \mathbf{H}_{3}\left(\tau_{k}\right) \boldsymbol{\Phi}_{T_{H V}}^{1 / 2} & \boldsymbol{\Phi}_{R_{H H}}^{1 / 2} \mathbf{H}_{4}\left(\tau_{k}\right) \boldsymbol{\Phi}_{T_{H H}}^{1 / 2}
\end{array}\right) \\
& \delta\left(\tau-\tau_{k}\right)
\end{aligned}
$$

In the above equation, the subscripts $V$ and $H$ refer to vertical and horizontal polarizations respectively, $P_{k}$ is the relative power of the channel arrival at delay $\tau_{k}, G_{p}$ is the channel propagation gain (inverse of path loss), and $G_{T}$ and $G_{R}$ are transmitter and receiver antenna directional gains (about $8 \mathrm{~dB}$ in the proposed system.). Matrices $\mathbf{H}_{1}\left(\tau_{k}\right), \mathbf{H}_{2}\left(\tau_{k}\right), \mathbf{H}_{3}\left(\tau_{k}\right), \mathbf{H}_{4}\left(\tau_{k}\right)$ in (16) have entries distributed as iid $\mathcal{C N}(0,1)$, generated independently for every delay $\tau_{k}$. An example of measured power delay profiles $P_{k}\left(\tau_{k}\right)$ is plotted in Fig. 6. It is recommended that the crosspolarization coupling factor $\eta \equiv 10^{-0.1 x_{p o l}}$, where $x_{p o l}$ is defined in (3), may be taken as $0.14\left(x_{p o l}=8.5 \mathrm{~dB}\right)$, the median value observed in Fig. 3. The correlation matrices for the two polarizations are generally different, and the scattering is also, in general, dependent on polarization. A reasonable assumption is that while the two polarizations have independent realizations in a scattering environment, they follow the same second order statistics. In such a case the correlation matrices may be generated for both polarizations using the same set of decay constants though different antenna locations in (13). The spatial correlations for every delay in (16) are in general different, as would correspond to the situation where particular delayed arrivals are associated with particular arrival angles. Because in this work the wideband SIMO and narrowband MIMO measurements were conducted separately, correlations as a function of delay cannot be obtained. One possibility is to assume the same correlations for all delays, which would be consistent with both narrowband and wideband measurements collected. Examples of such channels have been observed in some cellular urban channels, [34], where the azimuthdelay power spectrum $P(\phi, \tau)$ was found to be separable, $P(\phi, \tau)=P_{A}(\phi) P_{D}(\tau)$, in many cases. As spatial correlation is related to power angular spectrum through a Fourier transform, this implies the same correlation matrices for all delays.

\section{CONCLUSION}

An extensive campaign to characterize the wideband multiantenna radio propagation channel between two ground-based platforms was conducted in a rural area of Lakehurst, NJ. Dozens of links with ground level transmitter and receiver arrays were characterized in mixed wooded/open terrain over ranges of up to $4 \mathrm{~km}$. MIMO capacity was computed for an $8 \times 10$ MIMO system using the measured $\mathbf{H}$-matrices. It was found that median $8 \times 10$ MIMO capacity supported by the channel was about 5 times the corresponding $1 \times 1$ channel capacity, and 3 times the corresponding $1 \times 10$ capacity. It was found that using arrays containing antennas of both horizontal and vertical polarizations improves capacity for short range LOS channels by almost fifty percent. Rms delay spreads were found to have a median value of $0.6 \mu \mathrm{s}$, while the 90th percentile rms delay spread was $1.6 \mu$ s. Some key findings for the rural peer-to-peer channel as compared to the cellular channel may be noted:

Considerably larger spatial correlations (exponential decay of spatial correlation with a median characteristic constant on the order of $0.7 \mathrm{~m}^{-1}$ as compared to about $5 \mathrm{~m}^{-1}$ found for a mobile in Manhattan [4]). Narrower angular spread as suggested by the measured Doppler spectrum and spatial correlations. Shadow fading with far larger variation (standard deviation of $17.5 \mathrm{~dB}$ as compared to about $8 \mathrm{~dB}$ often reported in cellular measurements). Rms delay spread similar to that reported in cellular measurements with a median of about 0.6 $\mu$ s. Significant MIMO capacity, when antennas are spaced widely, offering about $80 \%$ of corresponding iid channel capacity. Similar capacities were observed in urban areas with smaller arrays. Higher cross polarization discrimination of about $8.5 \mathrm{~dB}$, yet overall benefit from use of dual polarization at modest SNRs.

\section{ACKNOWLEDGMENT}

Many thanks are due to Cuong Tran and Tod Sizer for providing the power amplifier and assisting with logistics, and Al Sauickie for building and installing the antenna mounts. We are also grateful to other participants for helping plan the experiment as well as participating in data gathering: Dennis Romain, Charles Villa, Jesse Cohen, Baron Brown, Marc Beacken, Craig Goetjen, Alex Pidwerbetsky, and Sean Saunders. We are indebted to Peter Wolniansky for designing and constructing the narrowband channel sounder, and wideband radios. Discussions with Constantinos Papadias, Dan Avidor, and Laurence Mailaender have improved this work, as well as comments from anonymous reviewers

\section{REFERENCES}

[1] C. Shen, C. Srisathapornphat, and C. Jaikaeo, "An adaptive management architecture for ad hoc networks," IEEE Commun. Mag., pp. 108-115, Feb. 2003.

[2] J. Freebersyser, "MNM project briefing," [Online]. Available: http://www.darpa.mil/ato/solicit/MNM/briefings/freebersyser.pdf

[3] G. J. Foschini and M. Gans, "On the limits of wireless communications in a fading environment when using multiple antennas," Wireless Pers. Commun., pp. 311-335, Mar. 1998.

[4] D. Chizhik, J. Ling, P. Wolniansky, R. Valenzuela, N. Costa, and K. Huber, "Multiple input multiple output measurements and modeling in Manhattan," IEEE J. Select Areas Commun., pp. 321-331, Apr. 2003.

[5] D. Porrat, P Kyritsi, and D. C. Cox , "MIMO capacity in hallways and adjacent rooms," in Proc. IEEE GLOBECOM, Nov. 2002, pp. 19301934.

[6] K. Sulonen, P. Suvikunnas, L. Vuokko, J. Kivinen, and P. Vainikainen, "Comparison of MIMO antenna configurations in picocell and microcell environments," IEEE J. Select. Areas Commun., pp. 703-712, June 2003.

[7] C. Martin, J. Winters, and N. Sollenberger, "Multiple-input multipleoutput (MIMO) radio channel measurements," in Proc. IEEE VTC-Fall, Sep. 2000, pp. 774-779.

[8] M. Gans, N. Amitay, Y. S.Yeh, H. Xu, T. C. Damen, R. A. Valenzuela, T. Sizer, R. Storz, D. Taylor, W. M. MacDonald, C. Tran, and A. Adamiecki, "Outdoor BLAST measurement system at $2.44 \mathrm{GHz}$ : Calibration and initial results," IEEE J. Select. Areas Commun., pp. 570-583, Apr. 2002.

[9] R. Hogg, J. McKean, and A. Craig, Introduction to Mathematical Statistics, 6th ed. Upper Saddle River, NJ: Prentice Hall, 2005, pg. 635.

[10] W. C.-Y. Lee and Y.-S. Yeh, "Polarization diversity system for mobile radio," IEEE Trans. Commun., pp. 912-923, Oct. 1972.

[11] D. Baum, D. Gore, R. Nabar, S. Panchanathan, K. V .S. Hari, V. Erceg, and A. J. Paulraj, "Measurement and characterization of broadband MIMO fixed wireless channels at $2.5 \mathrm{GHz}$," in Proc. IEEE ICWPC, Dec. 2000, pp. 203-206. 
[12] V. Erceg, L. J. Greenstein, S. Y. Tjandra, S. R. Parkoff, A. Gupta, B Kulic, A. A. Julius, and R. Bianchi, "An empirically based path loss model for wireless channels in suburban environments," IEEE J. Selected Areas Commun., pp. 1205-1211, July 1999.

[13] L. Greenstein, V. Erceg, Y S. Yeh, and M. Clark, "A new pathgain/delay-spread propagation model for digital cellular channels," IEEE Trans. Veh. Technol., pp. 477-485, May 1997.

[14] P. Pajusco, "Double characterizations of power angular spectrum in macrocell environment," IEE Electron. Lett., pp. 1565-1567, Oct. 2003.

[15] J. Wepman, J. Hoffman, and L. Loew, "Characterization of macrocellular PCS propagation channels in the 1850-1990 MHz Band," in Proc. IEEE ICUPC, Oct. 1994, pp. 165-170.

[16] E. Sousa, V. Jovanovic, and C. Daigneault, "Delay spread measurements for the digital cellular channel in Toronto," IEEE Trans. Veh. Technol., pp. 837-847, Nov. 1994

[17] P. A. Bello, "Correlation functions in a tapped delay line model of the orbital dipole channel," IEEE Trans. Inform. Theory, pp. 2-10, Jan. 1963.

[18] T. Zwick, D. Hampicke, J. Maurer, A. Richter, G. Sommerkorn, R. Thoma, and W. Wiesbeck, "Results of double-direction channel sounding measurements," in Proc. IEEE VTC-Spring, May 2000, pp. 24972501

[19] M. Steinbauer, A. Molisch, and E. Bonek, "The double directional radio channel," IEEE Antennas Propagat. Mag., pp. 51-63, Aug. 2001.

[20] D. Chizhik, G. Foschini, M. Gans, and R. Valenzeula, "Keyholes, correlations, and capacities of multielement transmit and receive antennas," IEEE Trans. Wireless Commun., pp. 361-368, Apr. 2002.

[21] D. Gesbert, H. Bolcskei, D. Gore, and A. Paulraj, "Outdoor MIMO wireless channels: Models and performance prediction," IEEE Trans. Commun., pp. 1926-1934, Dec. 2002.

[22] J. Ling, D. Chizhik, and R. Valenzuela, "Predicting multi-element receive \& transmit array capacity outdoors with ray-tracing," in Proc. IEEE VTC-Spring, May 2001, pp. 392-394.

[23] P. Kyritsi and D. Cox, "Modal analysis of MIMO capacity in a hallway," in Proc. IEEE Globecom, Nov. 2001, pp. 567-571.

[24] T. Aulin, "A modified model for the fading signal at a mobile radio channel," IEEE Trans. Veh. Technol., vol. 28, pp. 182-203, Aug. 1979.

[25] J. Andersen and K. Pedersen, "Angle of arrival statistics for low resolution antennas," IEEE Trans. Antennas Propagat., pp. 502-513, Mar. 2002

[26] K. Kalliola, H. Laitinen, P. Vainikainen, M. Toeltsch, J. Laurila, and E. Bonek, "3-D double direction radio channel characterization for urban macrocellular applications," IEEE Trans. Antennas Propagat., pp. 893897, Nov. 2003.

[27] C. Cheolhang, H. Bertoni, and G. Liang, "Monte Carlo simulation of delay and angle spread in different building environments," IEEE $J$. Select. Areas Commun., pp. 2191-2200, Nov. 2001.

[28] D.-S. Shiu, G. J. Foschini, M. J. Gans, and J. M.Kahn, "Fading correlation, and its effect on the capacity of multielement antenna systems," IEEE Trans. Commun., pp. 502-513, Mar. 2000.

[29] W. C. Jakes, Ed., Microwave Mobile Communications. New York: Wiley, 1974

[30] H. Xu, M. Gans, D. Chizhik, J. Ling, P. Wolniansky, and R. Valenzuela, "Spatial and temporal variations of MIMO channels and impacts on capactity," in Proc. IEEE ICC, Apr. 2002, pp. 262-266.

[31] K. I. Pedersen, J. B. Andersen, J. P. Kermoal, and P. Mogensen, "A stochastic multiple-input-multiple-output radio channel model for evaluation of space-time coding algorithms," in Proc. IEEE VTC-Fall, Sep. 2000, pp. 893-897.

[32] J. P. Kermoal, L. Schumacher, K. I. Pedersen, P. E. Mogensen, and F. Frederiksen, "A stochastic MIMO radio channel model with experimental validation," IEEE J. Select. Areas Commun., pp. 1211-1226, Aug. 2002

[33] D. Chizhik, F. Rashid-Farrokhi, J. Ling, and A. Lozano, "Effect of antenna separation on the capacity of BLAST in correlated channels," IEEE Commun. Lett., pp. 337-339, Nov. 2000.

[34] K. I. Pedersen, P. E. Mogensen, and B. H. Fleury, "A stochastic model of the temporal and azimuthal dispersion seen at the base station in outdoor propagation environments," IEEE Trans. Veh. Technol., vol. 49, pp. 437-447, Mar. 2000

[35] H. Özcelik, M. Herdin, W. Weichselberger, J. Wallace, and E. Bonek, "Deficiencies of the 'Kronecker' MIMO radio channel model," IEE Electron. Lett., pp. 1209-1210, Aug. 2003.
[36] C. Oestges and A. J.Paulraj, "Beneficial impact of channel correlations on MIMO capacity," IEE Electron. Lett., pp. 606-608, May 2004.

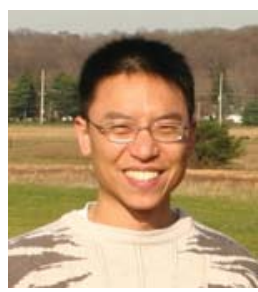

Jonathan Ling received the B.Sc degree in electrical engineering from Rutgers University, Piscataway, NJ in 1992, and the M.Sc. in computer science from Stevens Institute, Hoboken, NJ in 1999, where he is currently working toward a Ph.D. in electrical engineering. He joined the Wireless Communications Research Department of Bell Laboratories, Holmdel, NJ in 1994. He conducted radio propagation measurements and modeling in micro-cellular environments, and expanded the WiSE ray-tracing model to outdoor scenarios. His research interests are channel modeling and signal processing.

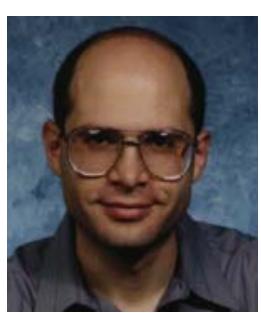

Dmitry Chizhik is a Member of Technical Staff in the Wireless Research Laboratory at Bell Labs, Lucent Technologies. He received a Ph.D. in Electrophysics at the Polytechnic University, Brooklyn, NY in 1991. His thesis work has been in ultrasonics and non-destructive evaluation. He joined the Naval Undersea Warfare Center, New London, CT where he did research in scattering from the ocean floor, geoacoustic modeling, and shallow water acoustic propagation. In 1996 he joined Bell Laboratories, working on radio propagation modeling and measurements, using deterministic and statistical techniques. His recent work has been in measurement, modeling, and channel estimation of MIMO channels. The results are used both for the determination of channel-imposed bounds on channel capacity and system performance, as well as for optimal antenna array design. His research interests are in acoustic and electromagnetic wave propagation, signal processing, and communications.

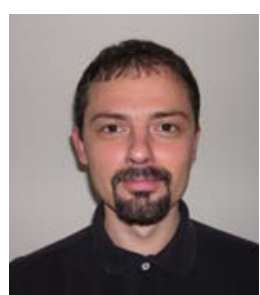

Dragan Samardzija was born in Kikinda, Serbia, in 1972. He received the B.S. degree in electrical engineering and computer science in 1996 from the University of Novi Sad, Serbia, and the M.S and $\mathrm{Ph} . \mathrm{D}$. degrees in electrical engineering from the Wireless Information Network Laboratory (WINLAB), Rutgers University, in 2000 and 2004, respectively. Since 2000 he has been with the Wireless Research Laboratory, Bell Laboratories, Alcatel Lucent, where he is involved in research in the field of MIMO wireless systems. His research interests include detection, estimation, and information theory for MIMO wireless systems, interference cancellation, and multiuser detection for multiple-access systems. He has also been focusing on the implementation aspects of various communication architectures and platforms.

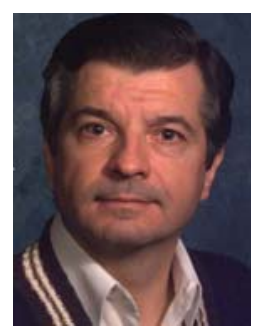

Reinaldo A. Valenzuela (LF'99) obtained his Bachelor of Science from the University of Chile and his Ph.D. from the Imperial College of Science and Technology of the University of London, England. At Bell Laboratories, he studied indoor microwave propagation and modeling, packet reservation multiple access for wireless systems, and optical WDM networks. He became Manager, Voice Research Dept., at Motorola Codex, involved in the implementation integrated voice and data packet systems. On returning to Bell Laboratories he led a multi-disciplinary team to create a software tool for Wireless System Engineering (WiSE), now in widespread use in Lucent Technologies. He received the Distinguished Member of Technical Staff award and is Director of the Wireless Communications Research Department. He is interested in microwave propagation measurements and models, intelligent antennas, third generation wireless systems, and space time systems achieving high capacities using transmit and receive antenna arrays. He has published over eighty papers and has twelve patents. He is a Fellow of the IEEE. He is editor for the IEEE TRANSACTIONS ON COMMUNICATIONS and the IEEE TRANSACTIONS ON WIRELESS COMMUNICATIONS. 